\title{
THE MIND AND THE THINKING MACHINE
}

\section{Eberhard Lammert $^{1}$}

"Cops all sit at computers nowadays. You and I are the last of the old breed. "This was what the policeman Rod Haines said in the 1985 movie Thompson's Last Run to the prisoner he was escorting, played brilliantly by Robert Mitchum. It must be said that Thompson is by no means an unwilling prisoner, though. Haines has much more faith in his own intelligence, a lifetime's experience and a good pair of handcuffs than in modern crime-fighting strategies, which have led to a goodly portion of police work taking place in front of the computer. However, when the safe-cracker Thompson escapes, neither one nor the other is any use, because the constellation of motives and opportunities that impells Thompson to make a break for it is entirely unforeseeable, and so conforms to no previous experience Haines has had, nor to anything programmed into a computer. Afterwards, of course, stubborn obstinacy and electronic Haines' technology both play their parts in temporarily recapturing the hero of this picaresque film.

In the course of the fugitive hunt, it is the computer - operated criminal - tracking techniques that bear the brunt of the burden in drawing the noose tighter. However, Thompson's arrest only comes about because of the police inspector's long

${ }^{1}$ Emeritus Professor of German and Comparative Literature, Free University of Berlin and Director of the Center for Literary Studies, Berlin; Former President of Free University of Berlin (1976-1983) acquaintanceship with Thompson over the years which had also given him an insight into the man's innermost motives. These are completely unrelated to his criminal activities and therefore cannot be played out even by the most sophisticated expert system. But Haines' store of knowledge, culled from his own memories of encounters with Thompson, permit him to make the association that puts him one jump ahead of the fugitive. Although this comes to his aid when all the technical wizardry fails, it is only this one time: an insight cannot be repeated at will.

Now, although Nietzsche has said that the knowledge that one has for oneself and shares with no one but a select few is the only really valuable knowledge, nonetheless it is not sufficient --as the case I have just described shows -- to make one a reliable authority in the broader spheres of human activity such as fighting crime or practising within an academic discipline. I can still remember the air of serene certainty with which an eminent scholar of classical philology declaimed his lines of Pindar or Cicero whenever we asked him about a lesser-known passage. "I have the entire corpus of my texts in my head," said he. The Nestor of classical philology, it was not solely his long memory that came to his aid, but also the fact that his cannon of texts was finite, while the number of colleagues he had and the amount of work they produced was manageable. Thus his scholarly competence could really be said to have reached its apogee. 
But it is just here that we can stop being guiltily, respectfully, envious: for all his knowledge could probably be recorded on a single CD Rom diskette, and the only obstacle in the way of any researcher in this field being able to make any connections or comparisons he may see fit is the patience of the programmer entrusted with the task of feeding in all possible search coordinates.

Already today, thanks to these expert systems, the Bible and the writings of Immanuel Kant are available to every school child. Yet in an age when the US Educational Testing Service informs us that all the research findings continuously being produced would fill an entire Encyclopedia Britannica every forty minutes or, as another source puts it, the knowledge we have today will have doubled within five years - in this age, in most fields, there is not the smallest chance of keeping in mind anything but a fragment of the knowledge necessary to be recognized as an authority in any field.

Throughout mankind's history already, since the development of hieroglyphics, cultures have always found ways of storing more knowledge than any individual can keep in memory; the library of Alexandria already contained enough to keep hundreds of readers occupied for a lifetime trying to read it all. However, a change has taken place which has led to a virtual explosion in the knowledge stored outside people's heads. Until the middle of this century, the goals our civilization had fixed for technology were essentially aimed at circumventing the necessity for hard manual labour, replacing it with machines or intellectual effort. The consequence has been that not only has the range of human activities in the domain of work undergone a significant transformation, but so also have the symptoms of work-related strain. The shift from physical labour to intellectual labour corresponds to the change from skin calluses to neurosis. With the rapid succession of computer generations and the subsequent leap to parallel processors, a tool has been created --even for intellectual operations--which enormously accelerates not only complex cognitive operations, but can also actually generate on command new and unforeseeable amounts of data.

In contrast to machines which produce energy for particular purposes or which transform material, the computer has the advantage of being universally applicable. This is due to the semiotic nature of computer programmes, and through its combinatory capacity, the computer entirely surpasses the reproduction attempts of all written languages, from hieroglyphics to sound imitation. Its binary sequences of ones and zeros can not only store information in different languages, but also, insofar as the interrelation can be formally expressed at all, can combine them. The universality of its sign system also explains why it can be introduced as a tool for everyone's intellectual work in all areas of life simultaneously, and in contrast to other radical innovations like nuclear energy or genetic engineering, which are restricted to the province of specialists--it can be extended "playfully" in every sense of the word into the pre-schoolers' domain. Thus it will become indispensable for department stores and for church administration, in legal proceedings and in medicine, and of course for nuclear energy and genetic engineering. 
However, it is chiefly in the race to solve problems in all areas of life which can be calculated that the computer is so much superior to all other devices invented by man. Already today, the doctor who does not use these superior diagnostic or testing technologies for difficult diagnoses or operations can be prosecuted--if he and his patients both happen to live in this fortunate part of the world.

Even more rapidly than it produces material goods and consumes energy, our growth-oriented culture generates a surplus of information. In fact, microprocessors and mainframe computers together now produce a million times more data than could ever be used in the time it takes to generate it. Years ago, Professor Harald Weinrich warned of the enormous effort required to scale the mountain of information and, should the summit ever be attained, of the melancholy outlook that awaits us there, when we can see only fog on all sides.

The knowledge that was a costly rarity almost until the beginning of this century, and which set scholars apart in the same way that medicine men had been privileged in earlier cultures, is now mass produced. It piles up day by day in electronic archives, and even gold would be worth only a fraction of what it is if it were available in these quantities; such is the incontrovertible law of production and demand. It is therefore appropriate that we should inquire into the nature of the knowledge that is conveyed to us in this way, spreading into more and more media and hoarded in ever more databases.

The road to modernity has, in Europe, been characterized by the replacement of a form of knowledge founded on generally accepted topoi, the World of the Bible and a fund of proverbs with a form of knowledge based on experience which, in conformity with its definition, can only be acquired through individual observation. Long before the inviolable rights of the individual had been enshrined in constitutions, this individualization of practical knowledge prepared the way for the atomization of individuals in modern societies. It became necessary to exert increasing effort to legitimize the general validity of this experiential knowledge anew with each succeeding generation. The new desire to share knowledge intersubjectively and thereby to objectivate gave rise in the sentimental climate of the eighteenth century to the cultivation of intense friendships developing into bonds between soul-mates. In the latter part of the age of industrialization, it led in turn to the rigorous privileging of measurable knowledge in respect to the legitimation of truths.

The information sciences confirmed to an unexpected degree the general trend, already envisioned by Leibniz, towards the mathematization of knowledge. Because this trend is obviously linked to the atomization of individuals in modernity, the continued vigorous assertion that the basic principle of the information sciences is communication is understandable. Yet in practice, work at the computer atomizes the user far more than any direct personal interaction in the office or in academic discourse (cf. Mumford, and in this regard, Nake, p.116). The computer, as the most sophisticated of calculators, fulfils in exchange the need for the intersubjective "alignment" of knowledge to a degree hitherto undreamt of. If there is any 
instrument at all capable of bridging the gap between the oft-procalimed two cultures of literary and technical intelligence, then it would have to be this machine, with its universal applicability.

But at what cost? The unambiguousness of command and execution is the thinking machine's great advantage when it is confronted with a task to be carried out. And if the programmers can only make available enough menus for every field of knowledge, and sufficient virtual realities for each one of us to be able to create his own mindgame, the individual use of general knowledge will be nearing an ideal state. In fact, in a volume of collected essays that came out in 1992, entitled Sichtweisen der Informatik, this development is described--in a carefully considered fashion--as a "continuation of evolution by other means." An increase in information and increases in complexity and acceleration are taken for--as they were in the past--the characteristics of former evolutions and so of this one which is actually taking place (Seetzen p.92).

Right away we have to ask what sort of data, bundled up as information, these more highly developed forms of humanity work with. If one considers thinking to be trial attempts at solving a problem, then it is indisputable that a computer user can expect, for every formalizable task, an enormous quantity of feasible solutions to be selected and tested. Such a quantity would be quite beyond his capacity to generate and achieve in the same time by any other means. With this capacity for serial testing, under certain circumstances a computer can run through a million combinations within a few minutes, in order to identify the right one. Nature would need thousands of years. In this regard, the "thinking machine" has indeed become an amplifier of our minds in the same way that the pneumatic hammer became the amplifier of our biceps.

When the mathematician Arthur Samuel developed a program for a game of checkers against which a human opponent had no chance of winning, he exploited just this advantage of serial testing of all calculable possibilities (Hofstadter, p. 644f). Any pessimism in regard to such examples should be dismissed immediately, though. Man has always invented tools to multiplicate his strength and thereby surpass the speed of his own legs or the load-bearing ability of his own shoulders. If he has now used his brain in the same way to create a multiplicator for his thinking energy, then he may regard all the achievements of artificial intelligence, even should they defeat him, as his own. It took nearly two thousand years before the seemingly everlasting Pons asinorum, the donkey's bridge, that Pappus needed to prove that the two opposite angles of a triangle with two equal sides were also equal, could be dispensed with. This says just as much for the programmer who was able to accomplish this feat as for the device whose technical and graphic capabilities he used in order to do it.

But do not let us deceive ourselves: these are accomplishments which can be plotted along a narrow path through the wide landscape of human thinking abilities, a track along which man, as programmer of his thinking, has progressed some little way. But scholars in particular are dutybound not to keep to the beaten track, but to strike out into the unknown. Just 
looking back along the path is really not enough.

The fact that its model for data processing could also immediately be employed in order to win new insights into the brain and the way it works, i.e. into cognitive processes, speaks for the new information sciences having hit a nerve in mankind. It was primarily the semiotic character that each object or concept had to take on when it was processed by computer that permitted the comparison between the computer's mode of functioning and the transformation of perception and stimuli into thought through a system of neurons. Significantly, this led to the notion, formulated in 1985 by Marvin Minsky, that during the working of the brain, each stimulus to thought must be transformed into a multitude of individual signals. It was only the comparison with the parallel processors necessary for accelerating computer processes that showed how enormously superior the achievements of the neuronal network in the human brain, as much its memory capacity as its ability to react, are to those of modern-day computer-systems. Jean-Francis Lyotard, who for this reason called "the thinking machines 'mentally subnormal,' even in comparison with a human brain which is out of practice" (p.820), will not necessarily always be right in this, for merely having the lead now in the unequal race between biological and technical development does not mean that one will win in the end. In any case, this consideration is enough to justify the assumption that learning processes in a machine governed by rules are comparable to those of a biological mental system.
A more decisive development was that information science cut itself loose from representation theory--even if it was two hundred years later than writers and artists had done--and declared information the real processor in every transformation of matter or energy. One does not even have to follow radical constructivism, holding that the product of sensual perception does not reproduce that which is given, but rather generates images, and thus also programs of action. One can understand without that the general validity the model of cognitive processes provided by information theory has for both machine remote-controlled and vital processes. Therefore it is not surprising that in the book just mentioned, Sichtweisen der Informatik, the Berlin systems analyst Jurgen Seetzen assures us that we need "no longer harbour any reservations about regarding information as the long sought-after vis vitalis, albeit on the basis of material and energetic phenomena and processes" (Seetzen, p.88). In the same way, it becomes evident why it was none other than information technology that provided the impulse for experiments or even mindgames that make it possible to abstract the complex neuronal processes of the human brain from their corporeal basis and to transpose them onto other frameworks. Therefore even assertions like "Communication alone communicates, not man" (Cf. Nake 1984, p. 116, on the origin of this thesis, Barck, p. 122ff.) no longer seem tautological or derogatory in regard to mankind. On the contrary, the notion of the spirit enjoying everlasting life in contrast to the body's mortality thereby acquires a new and much firmer foundation than it had in Neoplatonic ideas or in the Romantic notion of "elevated conversations of the spirits" between scholars throughout time. 
What was it about the thinking of a simple police inspector--Rod Haines--after Thompson's escape, that will not fit into the outline just described?

All the clever attempts to align the thinking capacity of machines with that of man are subjects to two restrictions which have not yet been mentioned: for one thing, they apply only in connection with relatively closed and therefore self-sustaining systems. In addition, moreover, these attempts apply only as long as they direct their attention uniquely at proving processes of a synonymous character, leaving aside all questions of substance and therewith substantive distinctions. This is a character trait that they share with the efforts at present of many scientific disciplines in the domain of cognitive theory--and admittedly, this limitation is the qualifying factor which offers safe passage in the transition from machine models to intercellular, macrobiological and social systems. For another thing, they leave out of consideration that which is lacking in every programmed process, yet which takes place unavoidably (and not always to the advantage of the one involved) in the mind of every human: and that is the continued and continual intermingling of deliberate thought processes with the quite incalculable store of day-to-day experience, both handed down through time and personal experience. The rather vague concept of 'day-to-day' is here meant to designate the inherently unorganized character of acquiring this experience as well as the elaboration of it. This was the constellation of experience and experiences that led police inspector Haines to the place the computer, fed on perfectly formulated criminological data, was unable to lead him to.

A few years ago. Francisco J. Varela drew a nice sketch comparing the tracks of hoofed animals in a fenced-in pasture with those in an unfenced one. The comparison speaks volumes. It makes it plausible that even Arthur Samuel's victory over himselfbeing defeated at checkers by his own computer program--is to be attributed to a world which, in this case, possesses the closure of a precisely defined crystal. In just the same way, every chess computer owes the extreme certainty of its operations to the finite world of possible situations which has been fed into it. With regard to robots, unexpected situations in the immediate environment require, even for "the simplest cognitive processes a seemingly infinitely large quantity of knowledge," which we "usually take tacitly for granted [...] but which must be fed into every computer spoon by spoon" (Varela 1990 , p. 93f.). Varela considers a robot driving a vehicle to be exposed to much greater danger than any well-behaved holder of a driver's licence, because of having to face the unforeseeable quantity of possible encounters even in a relatively well-regulated traffic system without the "limitless background of everyday knowledge." At the same time, even...the average driver of today can only trust his car if it has been fitted out with a whole range of electronic safety apparatus.

The recent evolution that Seetzen and also Mumford and Maturana prophesied is therefore based not on the replacement of man by machine, but rather on the wellregulated interplay between man and the machine invented to reduce his work-load. Of course, the temptation to rely too much 
on it poses a new threat. As tempting as it may sound the processing of information is not an imitative but a creative process, whether it takes place in a machine or in the mind, the difference between the programming of information channels--in principle circumscribed and regulated--and the limitless intermingling of larger areas of life, leads to narrowness of thinking. The tendency to seek the solution for difficult tasks mainly or even exclusively within the channels of thought offered by the programmed computer might even increase.

Now that not a few academics in the humanities are on the point of renouncing traditional hermeneutics and making the materially-linked nature of scientific and artistic operations responsible for their findings--just using the typewriter makes one think differently from how one did when using a goosequill--we must reckon with the possibility that the implementation of machine-oriented hardware and software, in the absence of any selfreflection, will at the very least no longer simply complement thinking but transform it qualitatively. In so doing, it will accustom us to taking formalizable thought operations fit for the computer for thinking as such. The procedure we use to determine whether our pre-med students are suited for studying medicine and later becoming doctors can already be regarded as a remarkably successful experiment in breeding this kind of thought. Ironically, it is called multiple choice, ${ }^{2}$ and it only permits the kind of question that can be formalized and answered only by 'yes'or 'no'.

2 Although it really, is known officially as 'multiple choice, we delight in calling it, more accurately, 'multiple guess'.
In reverse, however, our own form of thinking, which is 'wild' by comparison, cannot simply disregard the omnipresence of electronic information technology. The fact that thanks to the Internet connections we have at home and the multiplicity of telemedia, we live permanently "together with more information than we can process"--that is, into well-founded knowledge--induces the members of the socalled information society to believe information very much more than previous generations (Mittelstra $\beta 13$, pp. 6, 11).

The news, images and impressions which reach us daily via all types of media, from all parts of the world and all areas of knowledge, broaden exponentially the horizon of the reality thus conveyed, in contrast to that which one experiences or thinks out for oneself. From the increasing gap between systematically acquired knowledge and information offered by the media, Jurgen Mittelstra $\beta$ has drawn the conclusion that the prevailing attitude of dependence and the increasing acquisition of knowledge which by-passes experience will make knowledge indistinguishable from opinion. In a complicity intoxicated by pluralism, our freedom of opinion is menaced from a completely unexpected side by the danger of fundamentalism. The theory of consensus, underwritten by a concept that makes communication essential for all life processes, which brings truth and public opinion closer to each other, reinforces this trend at just the right moment. Transitional truths as the product of 'public opinion' are nothing new, not even in the juridical domain, but under the pressure of opinion exerted by mass media, the chances of their incontrovertible legitimation are raised by a qualitative leap. 
All this shows us that the processing of knowledge can no longer be the sole yardstick, or even the one which takes priority, for assessing the capability of a scholar or a scientist. In fact, since the inception of modernity, this ethos has made the search for new truths and their laws the most distinguished of duties, and the academic and scientific worlds and public opinion too have richly rewarded the discoverers of new laws of nature, new lands, new stars and also the rediscoverers of our past, the historians and archaeologists. It was not wrong to summarily call the modern age beginning with the discovery of America the Age of Discovery. If, in fact, the acquisition of knowledge today can be carried out serially, with a much higher degree of certainty, as if in a factory, and if on the other hand, the amount of information electronically sent or stored surpasses by far the amount that merits analysis or even examination, then that ideology of the new which gave us the wings to make the great discoveries of the modern age must itself be counterbalanced by an ability to judge that focuses on the purposes as well for which knowledge is accessed and applied in new cognitive operations.

It is precisely this sort of reflection - in dealing with information that can be accessed at will and with knowledge tested through experience - that can only take place in the mind, and the most a computer can do is check it. Quite apart from one's own fund of knowledge or professional competence, one uses previous life experience in order to assess the use that is to be made of this knowledge. This is that other knowledge that Varela somewhat incautiously referred to as "common sense" and which one could call, somewhat more cautiously, "the wisdom of life:" a wisdom which takes into consideration the life of other people as the condition of one's own life. Having recourse to this knowledge not already systematised renders it possible to assess the significance of a discovery for other spheres of life, and that with more certainty than the narrow perspective of expertise within a discipline. It is this very distance that allows the imagination more room to play, in order to understand the 'wit' of the knowledge so acquired. Information theory should welcome this recourse to the power of the imagination as processor for a sensitive assessment, because indeed, the giving of a form to something is closely related to imagining it. And the 'wit' lies in the fact that imagination and the memory cause to come together stores of knowledge which are otherwise unrelated. More even than in the German word "Witz" which John Locke and Christian Wolff used in the early eighteenth century to designate the capacity to make such surprising connections trespassing on professional boundaries, does the English word "wit" or our German expression "gewitzt" (which means "clever") remind us that "Witz" (mhd. "witzen") is etymologically the factitive verb and therefore the active variant of "knowledge."

More than any other discipline, cultural studies have reason enough today to reach consensus on such an active use of knowledge which takes in the 'wisdom of life' as well. The disciplines in the Faculty of Philosophy have, in contrast to what Kant and Wilhelm von Humboldt imagined, not sufficiently exploited their new place at the center of a reformed university to become the transmitter of ideas to all the faculties and at the same time, the authority 
for testing their practical knowledge. Instead of this, these disciplines once again enjoy draping themselves in the mantle concept of cultural studies, their departments distributed nicely between them and thus in the end developed into areas of extreme specialization themselves, which for the most part only allow to come to the fore their own disciplinary form of scholarliness. If it is true that only a healthy 'wit' exercised off the beaten track leads to unexpected new insights, then research, precisely in cultural studies, needs today more than ever interdisciplinarity and also teamwork in order to do justice to the radical shift in present-day and future demands. With the question as to how all the cultural studies should orient themselves in regard to the new writing and calculating and storage and search systems of artificial intelligence, the necessity of such interdisciplinarity hits all cultural studies particularly hard. Cultural studies - which to a major extent are historical and whose objects and forms of expression consist chiefly of coherent texts, naturally put up greater resistance to the introduction of rule-governed expert systems than those disciplines which are able to express their findings in formulae, statistics or units of measurement. Yet some terrains are indeed already staked out in the front yards of cultural studies. Nobody would want to do without the help of computers in editorial work or textual criticism, and the range of possible assistance has for a long time ranged from easy forms of counting up variations right up to the exciting attempts at author attribution through sufficiently numerous dimensions of text comparison. Thus a whole year's issues of The New York Times and all the other sources for Uwe Johnson's four volume Jahrestagen, which the untiring collectors of the Johnson Archive in Frankfurt have brought together on a single CD ROM diskette with the entire text of the novel, is still to be counted as one of the simpler aids. For this reason, I would like here to address the tensions that rule-governed 'text-processing' will necessarily give rise to in the hermeneutic disciplines in the course of their interpretative work.

We cannot speak about texts in any way without perceiving them from a particular perspective and with particular associations, which for their part are linked to epochs, generations, regions and social classes, but which for this very reason are not entirely determinative either in respect of degree or of quality. Therefore, in principle they are indescribable systematically and can be programmed only approximately. With the linear extension of a text, the range of its possible interpretations quickly increases exponentially, even if the langue of the interpretation sets clear limits. In a book which has just been published, Germanistik in der Mediengesellschaft, Burghard Rieger has described the obstacles which stand in the way of development programs for information processing systems consequent upon the variable constitution of meaning from texts (p. 379). As Lyotard has already done, he realizes that human superiority in adjusting to different interpretation intentions is overwhelming and that it in turn is based on the flexibility of the medium Sprache, which every living human being uses differently, not only in general, but in particular, as "parole" in every individual situation.

It is for this reason in no way surprising, but rather to be expected, that almost at the 
same time as we entered the Computer Age, none other than hermeneutics fell into disrepute as being at best a merely subjective work of knowledge, though possibly even dangerously fraught with ideology. It is just as logical that the rebellion of the sciences against hermeneutics, in view of the conspicuous leap from alphabetic writing to binary codes, brought the recording system onto the field. In the same breath it rapidly announces the end of the Gutenberg Galaxy, and energetically reminds us to reflect on those forms of acquisition of knowledge which are materially linked and which are responsible in the different epochs of our history, as much for the specific possibilities as for the boundaries of knowledge and the mediation of knowledge.

There is no doubt about it, epistemological theory and cultural history as the history of technical mastery of life are entering into a new pact which poses an extremely stimulating challenge for all hermeneutical sciences. The reversal is already being spoken of, that "the materiality of communication is primarily a materiality of its media" (Schulte-Sasse, p. 433). If this hypothesis has any likelihood at all in view of the rabbit-like proliferation of personal computers in our libraries, on our writing desks and in our children's rooms, then it is precisely the historians of culture more than other academics who are the right people to uncover both new opportunities and also the new limits of scientific communication in this stage of our civilisation. It is they, much more than today's specialists of social sciences, because they can easily inspect several stages of writing culture comparatively across the diachronic range of their objects.
A sidelong glance at the seismographic achievements of art is called for. Already, at the beginning of our century and more or less at the same time as the cinematographic technology which taught pictures to run and began to portray the world discontinuously to the observer in cut and cross-cut composers of the avant-garde had begun to replace tonal sequences with free, and only immanently structured sequences of notes; already painters had begun to represent faces from the front and in profile at the same time, western European and American writers had begun to refine the technique of the "shifting view point" into a technique of describing simultaneous events or into "mise en ab îme" which are in stark contrast with linear reading. Not only did these experimenters want to offer the human imagination more to see and hear, but above all, they wanted to offer it different modes of experience. In his essay on Leskow, Walter Benjamin has already convincingly described that which is lost thereby, the peaceful pursuit of a narrative and trust in the narrator choosing the most important and the most significant things to recount.

It was not only until sixty years later " 'Tis is sixty years since"-that computer technology matured enough to provide us with texts that can be read not only line by line, but also with hypertexts which can 'deepen,' add to, surround the primary text through as many windows as one wants, opening out on all sides - and more importantly, can alter and shift that same primary text. Electronic 'books' are on the way to having us read even the past from a multitude of perspectives - for example, the discovery and colonization of America. In practice, it looks something like this: for a series entitled The Discovery of America, 
first a menu with a variety of lead-ins appears on the screen, and then, after clicking on any rubric, various other windows. After opening one of these windows, one sees a battered scribbler lying open, and underneath the words "Log Book" and a bit of text that describes a landing. Next to this is a contemporary engraving: a Portuguese grandee steps onto a palm-fringed beach, accompanied by his entire entourage, ceremonially dressed, but heavily armed. A little distance way is a group of indigenous inhabitants - almost naked. Under the engraving its provenance is given. Clicking back another window with the title "Yields" can be opened. When the window is opened, there is a list describing a ship's cargo, and next to it, a depiction of the arrival of a sailing ship in the harbour of Lisbon. This is accompanied by a voice-over which enumerates the size of the ship and its crew and then the goods which were disembarked. Another window in turn presents, when opened, that contemporary engraving with the landing of the Portuguese, but converted to another perspective with the aid of a kind of cyberspace technique: the observer looks over the shoulders of the natives, who now stand in the foreground. Underneath we read "The first Indian who discovered a European made a bad discovery." To the right, underneath this, in small print: "Georg Christoph Lichtenberg, 1742 1799."

Admittedly a simple lesson. But we learn from this that knowledge about the discovery of America, whether illustrated or not, can no longer be created page by page from a linear text whose sequence and elaboration is logically thought out by an author, but instead is selected and arranged, composed into a mosaic.
Although it is multimedia, it can just as well present as omit information: monotonous material or even, with a stroke of luck, contradictory - all according to how one moves the mouse. It is much more important that all succeeding users can also delete or add, so that a Hypertext is created simultaneously in several respects: it is an open-ended text, a text which, on the screen, can always complete, cut back or even change the foregoing one.

We are on the way to returning to the scholars of the middle ages, when every copyist could shift around, omit or exchange passages in a manuscript written by multiple copyists, in order to fit new purposes - or just by mistake. From now on, every reader can step into the role of the mediaeval copyist, the only difference being that his behaviour is no longer determined by biblical topoi and worldly proverbs as a general counterbalance to his or her individual passions. Even so, however, it is only afterward that this new freedom casts a light on the hidden constraint of causality which printed texts, for centuries unchangeable, imposed on reading.

Burghard Rieger draws three conclusions regarding working with such multidimensional hyperdocumentation:

1) The distinction between author and reader of a text is no longer necessary; no matter how it is created, the text in its contemporary form has no author-subject any more.

2) Its users can not only insert commentaries to the individual key words in accordance with their experience and the understanding they bring to them, but also change interrelations. It can thereby rapidly become difficult to get an overview 
of hyperstructures in their depth and organization.

3) Even when only one user works on a hyperdocument in a single subject area, without the necessity for linear logic, this can "very quickly reach a degree of complexity that makes it difficult for him to use his hypertext system fruitfully without help in navigation." (p. 393).

Perhaps my example has become too simple due to its brevity. But it should have become evident that the difficulty in orientation of which early twentieth-century art, with the simultaneous stage, the shifting viewpoint and the simultaneously full-face and profile representations already gave us a presentiment, is just about to become normal practice in daily life for everyone. The admirable storage, combinatory and generative capacity of computers will plunge us into an orientative twilight. Although it gives the individual a degree of freedom hitherto unmatched, in view of the pluridimensionality, of the text, the individual is more than ever in need of a navigator or a control program, no less than a pilot is, in order to deal with the infinity of electronically elaborated information now available. We have not yet even touched upon the issue of the individual producing combinations of erratic elements, as collage techniques in art have long since shown.

However, even the extensive "erosion of subject-centred knowledge" (Schulte-Sasse, p. 441) down to an automatic - and in some cases also autopoietic--ludic conglomerate cannot prevent this way particular structures of knowledge being one-sidedly cultivated. For example, there are those that no longer accord particular significance to the causal connection of the material presented, nor to the finality of the learning process; by following this route, the indisputably positive motivation to action on the part of the user and also the stimulation of his creativity could well remain devoid of goals or purpose.

This substantiates the finding that easier access to information that may be called up at will and which, at the same time, is hardly directed any more requires a complementary increase in discriminatory capabilities. It is becoming more and more urgent that wisdom be deployed in the disposition of the ever more collected knowledge being passed on. The assessment of the consequences of the technology definitely does not remain a task for engineering or the natural sciences --quite the contrary, it is precisely cultural studies that, by researching the conventions of the acquisition of knowledge and its "recording systems" put themselves in the position to found such claims logically and historically.

One task of the disciplines of culture understood in this way, a task which goes far beyond simply an assessment of the consequences of the transformation of our writing culture, consists in keeping an open mind for different practices of transmission of the past, not cuddling up comfortably to old habits, but rather keeping open, in a comparative mode, the particularities and the limits too of our present opportunities for knowledge. In order to distinguish not only right from wrong, but also important from unimportant within these limits, additional distinctions of value are indeed necessary, and these no computer can give.

The university is a place for individuals and faculties to discuss such distinctions of 
merit in regard to continually enriched knowledge. It is only in the exchange of expert knowledge painstakingly acquired that such - possibly competing - value assessments are to be made. It is precisely when it becomes clear that such value decisions are not to be brought about with scientific arguments alone - it being much more arguments in regard to the world we live in, i.e. political, social, economic and even arguments coloured by cast of mind that usually take pride of place - that the university as a whole can again become, the site of an interdisciplinarily oriented reflection.

While the process technologist Gunter Spur, who is Rector of the Technische Universität Cottbus at the moment, still hopes that computer technology will liberate future teachers to a large degree from the conveying of knowledge, allowing them to function much more as educators and consultants for the organizing and the analysis of this knowledge on call, Erich Kiefer, the author of the books, about metacognition and virtual reality also promises, last but not least, intelligent learning systems which will "take over that function in the transmission of knowledge which, from primary school to university, is still fulfilled by teachers" (p. 209). They will be quick and efficient in the end, but they should only be allowed to make the move from being a work instrument to domination the stage when we do not know any more what we should use our minds for.

It is never possible to foresee clearly whether the inventions that people promise themselves will relieve their work-load and at the same time increase their power will be a blessing to mankind or the ruin of it.
It is for this very reason that the mind, after handing over a helping hand and its thought processes to machines is still dogged by worry. It is incumbent upon man to keep watch uninterruptedly over the mistakes which, due to his natural imperfection, are the possible dowry of all dealing and which, without machines, remain only his own, but which - transposed to machines can exponentially increase their effects, irrespective of the relief they bring him in relieving him of work.

Goethe prepares for his blind Faust a last and deepest humbling before his redemption. He allows him to neglect to take heed of anything. Thus deluded, Faust digs his own grave by a putatively gigantic feat of engineering. A computer story of only a few lines--entitled Grips and Chips --which Karl Markus Michel culled from a British collection of riddles of logic sends a reminder about keeping alive this human care through continual reflection on the high-handedness of a self-generated machine world. I too would like to close with this story:

When the world's first fully automated airplane took off, the passengers were a little worried. Then they heard over the intercom the quiet, calming voice of the computer: "Ladies and gentlemen, you are enjoying the privilege of flying in the world's first fully automated airplane. There are no pilots who could make any human errors; you are being steered by computers which are error-free. All your needs will be taken care of. You have no need to worry about--to worry about--to worry about-- ...." 